\title{
Improving the modelling of the geomagnetic main-field: Isolating the average ionospheric field in satellite data
}

\author{
A. W. P. Thomson \\ Global Seismology and Geomagnetism Group, British Geological Survey, West Mains Road, Edinburgh, EH9 3LA, Scotland, U.K.
}

(Received February 14, 2000; Revised July 3, 2000; Accepted July 12, 2000)

\begin{abstract}
Olsen's method (Olsen, 1996) is applied to Magsat magnetic field data to deduce average quiet-time and activetime ionospheric current systems at middle to high North and South latitudes. Spot magnetic field values at ground level are calculated from the average models and are 'ground-truthed' against observatory data. The results suggest that synoptic ionospheric models from Magsat-quality satellite data may be helpful in reducing the contamination of the main-field by non-field-aligned ionospheric sources. We discuss how the technique may be applied to data from the Ørsted mission and the extent to which these ionospheric field models may therefore be useful in improved modelling of the geomagnetic main field.
\end{abstract}

\section{Introduction}

Models of the geomagnetic main, that is core-generated, field are traditionally derived from a wide variety of measured magnetic data (e.g. Langel, 1987; Quinn et al., 1997). It is also customary practice to pre-filter these data to remove, or 'clean', measurements made during active magnetic conditions. Surface magnetic field measurements can be treated in such a way that they can be considered largely free of ionospheric and magnetospheric fields, for example, by averaging observations under quiet external conditions (e.g. Quinn et al., 1997; Thomson et al., 1997). With satellite measurements however, the satellite may pass closer to or through, the source environments of the ionospheric and external fields and care needs to be exercised in treating these data satisfactorily. Again, pre-filtering according to activity level (e.g. Dst, $K p$ and $A E$ magnetic activity indices), as well as local time (LT), magnetic local time (MLT) and solar zenith angle is common (e.g. Langel and Hinze, 1998). Rejection of satellite data from the auroral and polar latitudes, perhaps depending on the external radiation level in the immediate environment of the satellite, or when the interplanetary magnetic field (IMF) is Southwards, is also a possibility. However even during magnetically quiet periods there will remain ionospheric and magnetospheric contributions to the measured field at satellite altitudes.

In this paper we examine whether a relatively simple approach to the problem of isolating the contributions of these other fields might help to improve models of the main geomagnetic field. The relative simplicity of our approach, it is noted, contrasts with the more complex ionospheric modelling techniques of, for example, Kisabeth and Rostoker (1977), Kamide and Matsushita (1979a, b), Kamide et al. (1981), Rich and Kamide (1983) and Walker et al. (1997).
The results presented here are regarded as an initial step towards exploiting high quality data from future satellite missions, of which Ørsted is the first.

Main-field modelling requires good data coverage over the Earth and, given the sample rates typical of satellite instruments, this usually implies the accumulation of large data sets. Any treatment of satellite magnetic data to remove ionospheric and external currents effects must therefore be both simple and straightforward enough that these quantities of measured data can be readily processed. The method introduced by Olsen (1996) is a useful technique in this regard, being computationally undemanding. It is also useful insofar as only satellite data are required to determine the ionospheric currents rather than ground-based data, for which there may be uncertainties in crustal field contributions.

Olsen's method is briefly described in the Appendix, as is the way in which the method is used here to derive synoptic ionospheric current maps. Below, we present average quiet-time and active-time ionospheric maps and data for particular seasons during the Magsat mission. The relative accuracy of these maps, in terms of reproducing the measured Magsat data set, is discussed. The ionospheric models are then used to provide spot values at a number of Northern hemisphere observatories and these values are compared with observatory hourly mean perturbations. This allows us to 'ground-truth' the data, i.e. check the quality and accuracy of the ionospheric models. We conclude that there is a reasonable agreement between the synoptic models and the ionospheric perturbations recorded at the observatories and that these models therefore represent useful data (or constraints) in main-field modelling. Finally, we discuss the potential applications of the method to the anticipated Ørsted data set. 


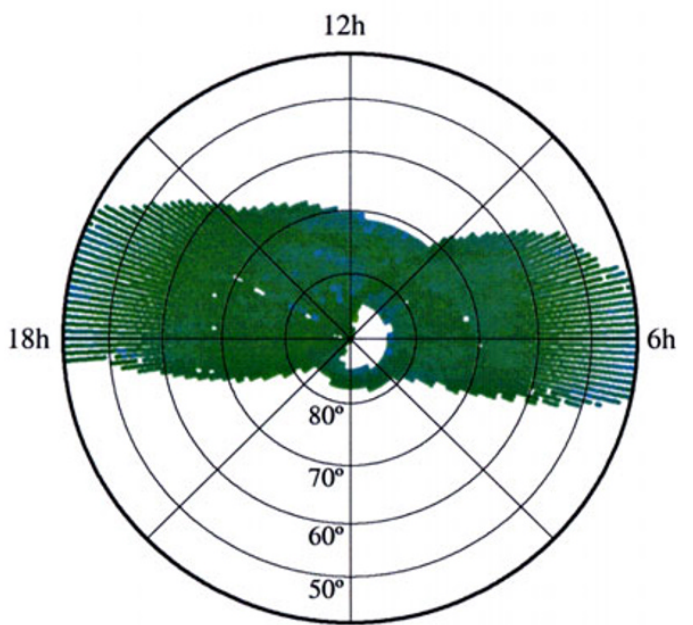

oh

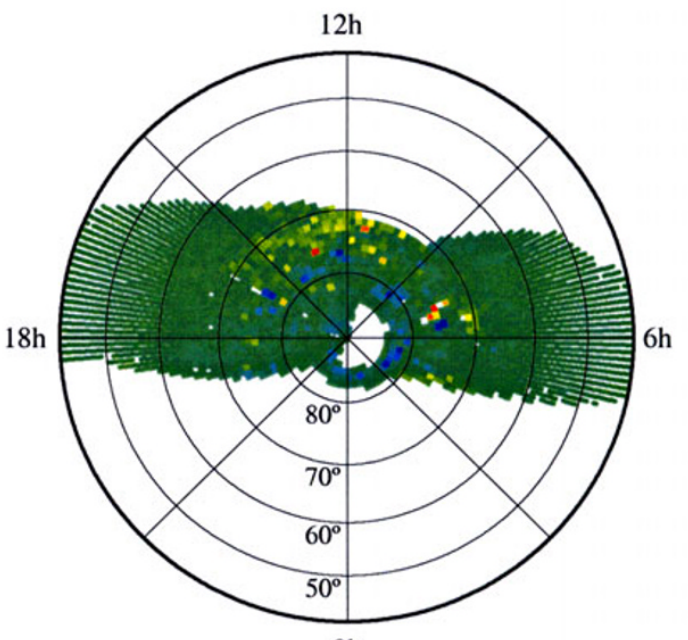

Oh

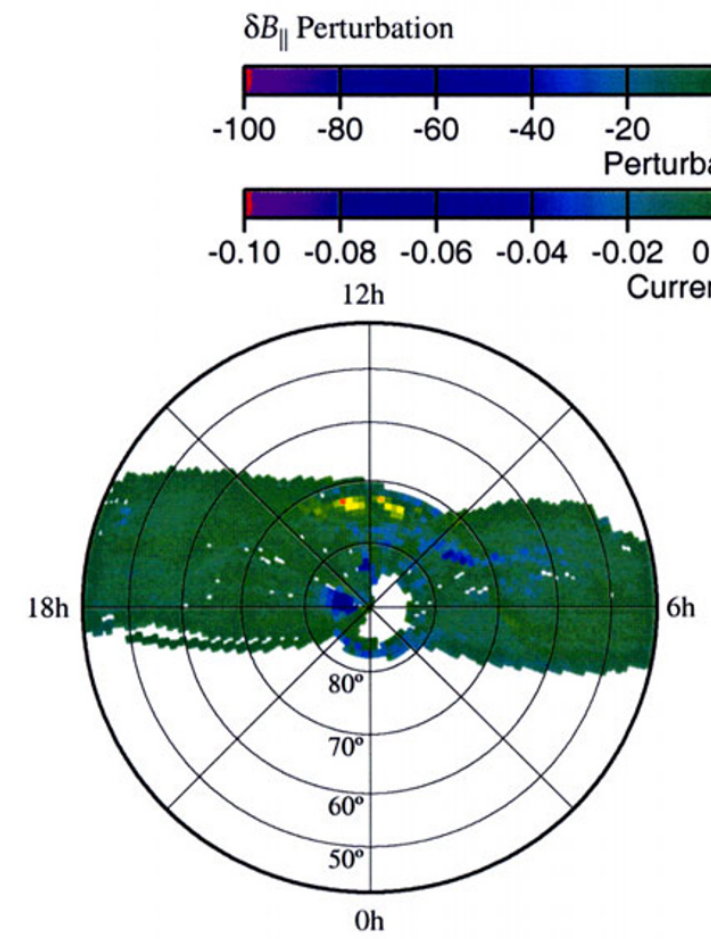

Dipole North Current
$6 \mathrm{~h} \quad 18 \mathrm{~h}$

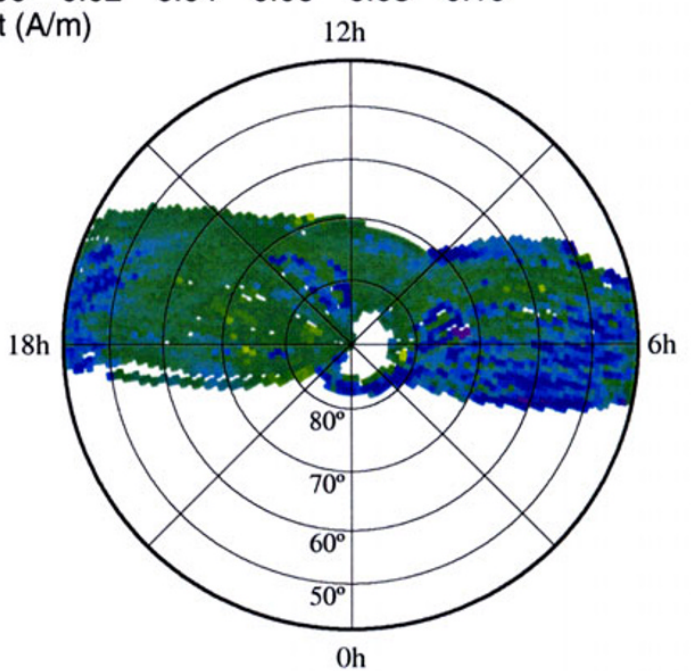

Dipole East Current

Fig. 1. Measured field perturbations and computed currents for the Northern hemisphere winter season under quiet magnetic field conditions. Co-ordinates are dipole latitude and magnetic local time. Maps are constructed from the 147 'quiet' Magsat orbits during December 1979 and January 1980. Field perturbations and currents are averaged in equal area tesserae, equivalent to $1^{\circ} \times 1^{\circ}$ at the equator (see Appendix). Quiet magnetic field conditions are defined as $K p \leq 1_{+},|D s t| \leq 25, A E \leq 50$.

\section{Quiet-Time and Active-Time Magsat Iono- spheric Models}

An example of a quiet-time ionospheric map is shown in Fig. 1 for the Northern Winter period of 1 st December 1979 to 31st January 1980. Within this figure both tessera-averaged field perturbations and the derived, tessera-averaged, currents are shown in a dipole-latitude and MLT coordinate system. (See Appendix for more details on the construction of these maps.) In Fig. 2 we show the active-time map for the same period. Quiet- and active-time conditions are defined, broadly speaking, as $K p \leq 1_{+}$and $K p \geq 4$ respectively (but see Figure Captions). Note that the 'holes' in the cover- age, particularly round the poles, are the result of the small tesseral dimension and the satellite orbital inclination.

In Fig. 1, it is seen that the perturbation parallel to the main field, $\delta B_{\|}$, is typically smaller than that of $\delta B_{\perp}$. There is also some indication of both the field perturbations and equivalent currents being organised in dipole latitude and MLT, even under these quiet conditions. The northern component of the ionospheric current is smaller than that of the eastern component, as a result of the orientation of the main current systems and the orbital path of Magsat. Similar features are seen in the Southern hemisphere map (not shown here). In particular, the perturbations and currents in the Southern 


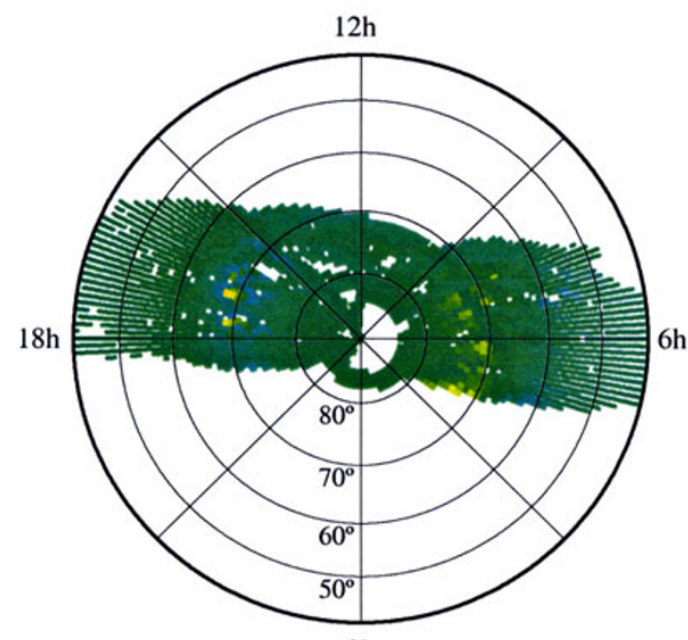

$\mathrm{Oh}$

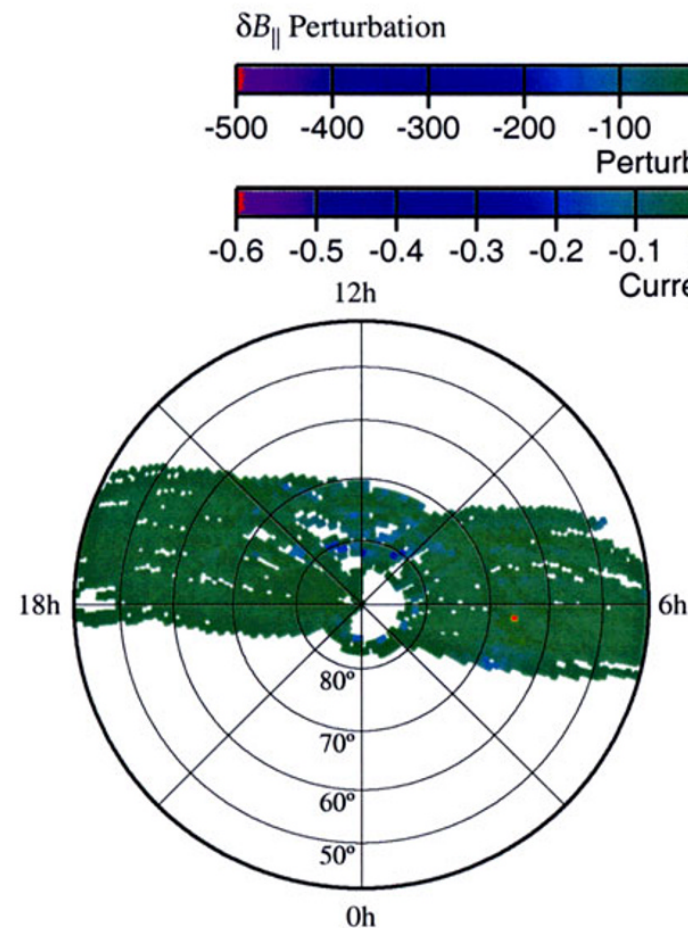

Dipole North Current

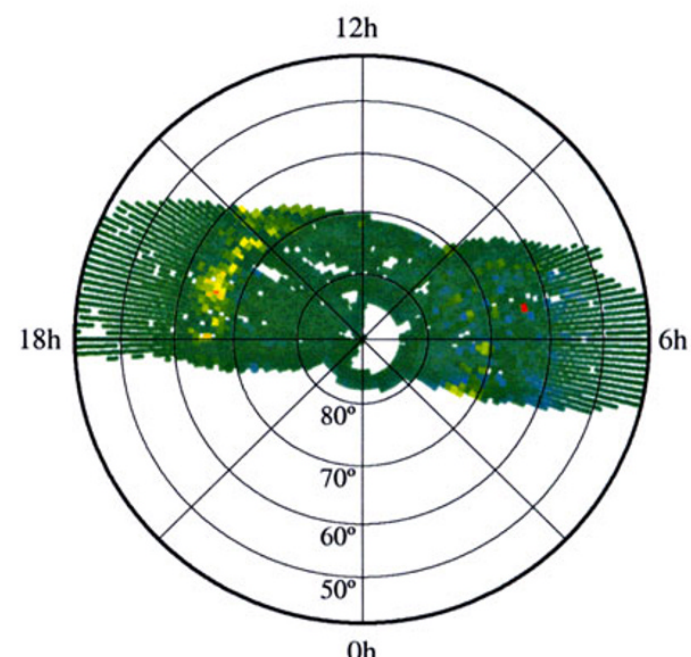

$\delta B_{\downarrow}$ Perturbation
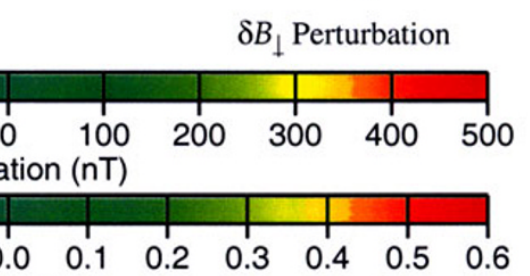

$(\mathrm{A} / \mathrm{m})$
$6 \mathrm{~h}$

$18 \mathrm{~h}$

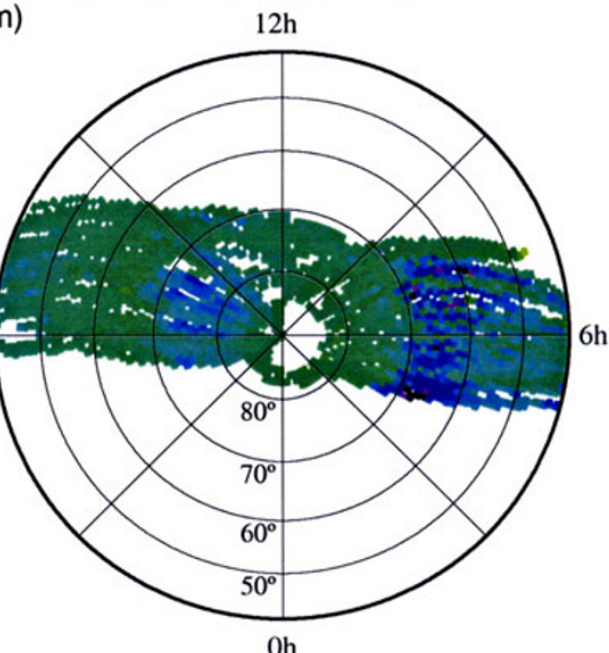

$\mathrm{Oh}$

Dipole East Current

Fig. 2. As Fig. 1 for the Northern hemisphere winter season, under active magnetic field conditions and from the 58 'active' Magsat orbits during December 1979 and January 1980. Active conditions are defined as $K p \geq 4$, with no filtering on Dst and $A E$.

map are clearly enhanced in this more sunlit hemisphere, compared to the Winter conditions applicable in Fig. 1.

Similar maps have been produced for the equinox periods, by using data from March and April 1980 to produce Northern Spring and Southern Autumn maps. These are also not shown here for brevity. As a general rule, the maps are similar in structure to Fig. 1 and its corresponding Southern hemisphere map. However we observe an enhancement, compared to Fig. 1, in the magnitudes of the perturbations and currents during the transition from Northern hemisphere Winter to Spring. On the other hand, there is a diminution in the magnitudes of the perturbations and currents in the transition between the Southern hemisphere Summer and Autumn. Both these results are to be expected.
Figure 2 shows enhanced perturbations and current vectors, with respect to Fig. 1, during more active intervals. The dipole co-latitude of the main arc of East-West currents increases by around $10^{\circ}$. The morphology of the currents can also be seen to change from Fig. 1. In particular there is a stronger 'banding' of the dipole-East currents in latitude and less variability with MLT.

Figures 1 and 2 here (i.e. quiet and disturbed magnetic conditions) compare with, for example, figures 1(c) and 2(c) of Kamide et al. (1981), under similar magnetic conditions, but there deduced solely from ground-based data. It is difficult to readily make comparisons with our data and that of Kamide et al., because of the different presentation styles. However we note the following. The equivalent current data 
Table 1. Accuracy of ionospheric models based on exact currents (EC), i.e. from the model solution, and tessera-averaged currents (AC). Values are field perturbations - mean and standard deviation in the mean, in nT-measured along all orbits in each sample. Filters are defined in the captions to Figs. 1 and 2 .

\begin{tabular}{|c|c|c|c|c|c|c|c|c|}
\hline \multirow{2}{*}{$\begin{array}{l}\text { Hemisphere, Season and } \\
\text { Activity Filter }\end{array}$} & \multicolumn{2}{|c|}{$\delta B_{\|}(\mathrm{EC})$} & \multicolumn{2}{|c|}{$\delta B_{\|}(\mathrm{AC})$} & \multicolumn{2}{|c|}{$\delta B_{\perp}(\mathrm{EC})$} & \multicolumn{2}{|c|}{$\delta B_{\perp}(\mathrm{AC})$} \\
\hline & Mean error & $\mathrm{SD}$ & Mean error & SD & Mean error & $\mathrm{SD}$ & Mean error & SD \\
\hline Northern: Winter: Quiet & 0.3 & 1.1 & 0.6 & 6.9 & 17.1 & 24.4 & 16.4 & 21.8 \\
\hline Northern: Winter: Active & 1.9 & 3.0 & 3.9 & 24.9 & 42.6 & 91.2 & 39.8 & 89.3 \\
\hline Northern: Spring: Quiet & -1.8 & 1.6 & -2.6 & 9.6 & 9.1 & 34.6 & 9.5 & 32.8 \\
\hline Northern: Spring: Active & -3.6 & 3.7 & -4.0 & 36.4 & 52.8 & 142.4 & 53.2 & 134.1 \\
\hline Southern: Summer: Quiet & 4.7 & 5.0 & 7.0 & 15.6 & 7.1 & 48.5 & 7.3 & 44.8 \\
\hline Southern: Summer: Active & 10.9 & 12.4 & 16.8 & 46.9 & 18.9 & 115.2 & 19.1 & 111.4 \\
\hline Southern: Autumn: Quiet & 4.2 & 4.0 & 6.2 & 8.3 & 7.5 & 26.4 & 6.9 & 21.9 \\
\hline Southern: Autumn: Active & 12.9 & 9.9 & 15.3 & 43.7 & 1.1 & 98.5 & 1.0 & 96.6 \\
\hline
\end{tabular}

of Kamide et al. seem to show most morphological change between quiet and disturbed conditions on the high-latitude day-side and these data also differ most from our results in that sector (although maximum current amplitudes in $\mathrm{A} / \mathrm{m}$ are comparable). On the night-side there is an equator-ward enhancement of the east-west electrojet, but probably not as much as the $10^{\circ}$ as is suggested here. The agreement between this work and that of Kamide et al. is possibly closest under the more active conditions on the night-side and away from the polar cap. This may be at least partly because quiet ground-based data may be more affected by uncertainties in the estimated crustal field. In addition, the data of Kamide et al. were collected under Northern hemisphere summer, unlike here. On the other hand, Figs. 1 and 2 show that the observed perturbations, $\delta B_{\|}$and $\delta B_{\perp}$, are similar to that of the map of the radial perturbation $\delta B_{r}$ in Langel and Hinze (1998, figure 4.19).

To examine how well the averaged currents reproduce observed field perturbations, in Table 1 we compare the mean error and the standard deviation in the mean error (SD) between the measured and calculated magnetic perturbations, over all data in each hemisphere. Table 1 demonstrates the impact of replacing the exact current (EC) solutions (i.e. best fit) for each orbit with the tessera-average current (AC) systems of Figs. 1 and 2 and their equivalents for the Southern Hemisphere. We also list in Table 1 the accuracy of the other ionospheric models we have constructed, for the Northern and Southern hemispheres during the March/April season.

By EC in Table 1 we mean the following. For each Magsat orbit we calculate the perturbations along each orbit from the best-fit currents for that particular orbit. Tesseral averages of these perturbations are then calculated from all the Magsat orbits in the sample. By AC we mean, in contrast, that a tesseral-average current system is used to calculate the perturbations along each individual orbit. In essence, for $\mathrm{AC}$, the best-fit currents, for each orbit, are replaced by their tesseral-average equivalents.

In principle the $\mathrm{AC}$ solution should be a poorer fit, compared to the optimum EC solution. Table 1 shows this and also demonstrates the trends with season and activity level noted above. For example, the use of averaged currents can be seen to degrade the $\mathrm{SD}$ in the fit to $\delta B_{\|}$(i.e. compared to the fit from the exact currents for each orbit) by a factor between about 2 and 10, depending on activity level, hemisphere and season. For $\delta B_{\perp}$, however, there is little change in the accuracy level between EC and AC maps, probably reflecting the greater contribution from field-aligned currents to the perturbations in this component (Olsen, 1996). It is seen that the $\mathrm{SD}$ in the $\mathrm{AC} \delta B_{\perp}$ is always smaller than in the corresponding EC perturbations, though this is not obviously significant.

\section{Ionospheric Model 'Ground-Truth'}

Although we have shown that it is possible to obtain reasonable agreement between modelled and measured satellite magnetic perturbations, it is important to check the consistency of these models with reference to an independent source. For this we have used the network of Northern hemisphere geomagnetic observatories shown in Fig. 3. This provides a wide range in latitude and longitude, particularly with reference to the auroral oval. Digital hourly mean data are available for all of these observatories. By subtracting a main field and crustal field estimate from these data we have constructed hourly-mean perturbations at each observatory, assumed to be due to external currents.

In Figs. 4 and 5 we compare model spot values calculated for the observatories shown in Fig. 3 with the average of the observatory hourly-mean perturbations in January 1980, in afternoon and morning local time sectors respectively, and under active magnetic conditions (see Figure Captions for further details). Like the observatory perturbations, the Magsat data represent average field perturbations due to equivalent ionospheric currents. We use active conditions here to demonstrate that the method is useful under such conditions and also because quiet condition perturbation data for observatories may be relatively affected more strongly by inaccurate crustal bias correction.

Model spot values are calculated from the tessera-averaged currents along actual Magsat flight lines within $\pm 2.5^{\circ}$ in longitude of each observatory. These tracks are then effectively 'overlain' on the ionospheric maps to specify the average currents at $1^{\circ}$-latitude intervals. Each track therefore gives a single estimate of the magnetic perturbation at the observatory. Since there are generally a number of such orbits 


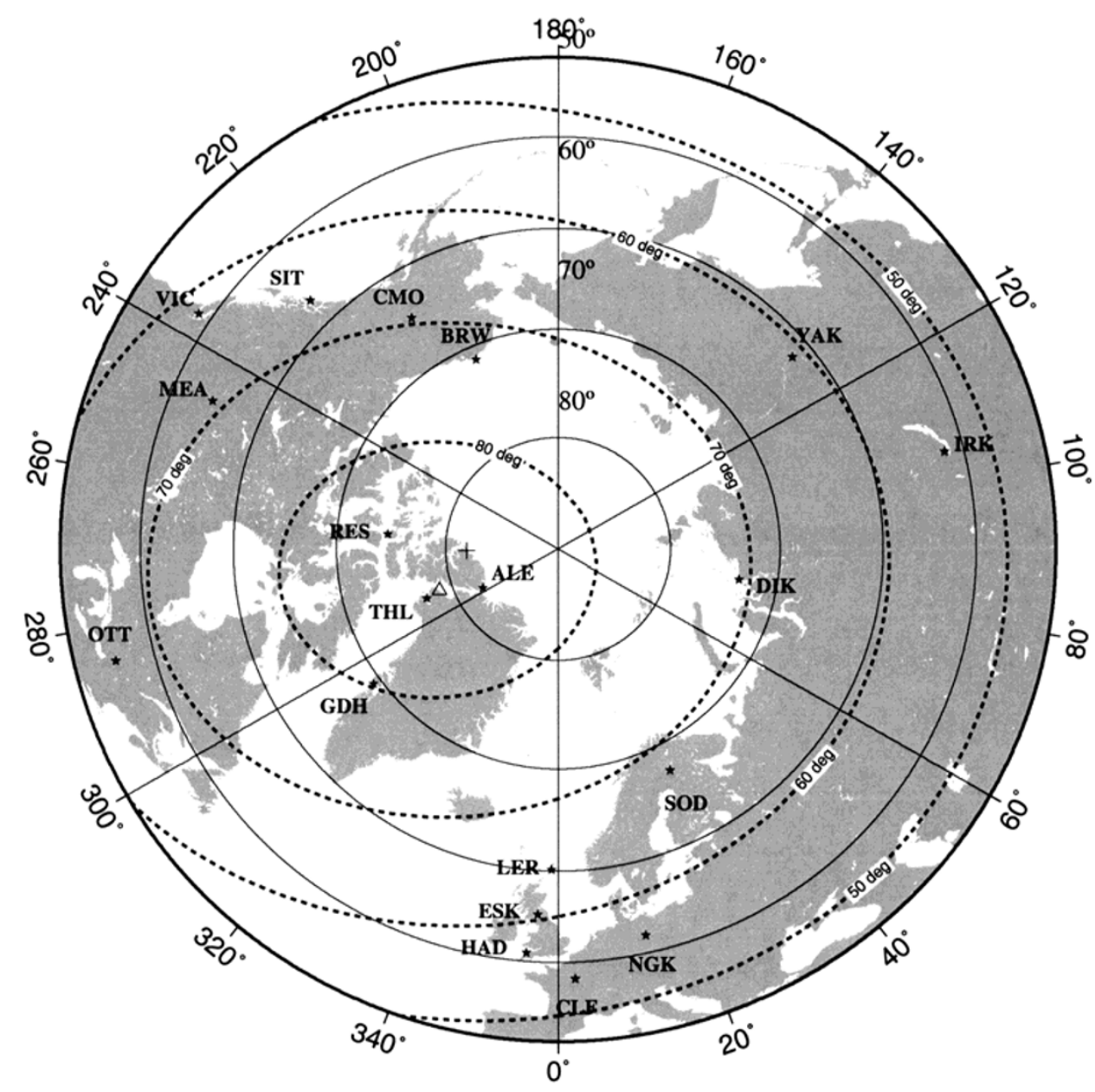

Fig. 3. Locations of Northern hemisphere observatories used in the 'ground-truth' study. The triangle and cross mark the locations of the dipole and eccentric-dipole pole at 1980.0. Standard IAGA three-letter codes for the observatories are shown, as are (dashed) contours of corrected geomagnetic latitude for zero altitude.

(depending on the latitude of the observatory) the spot value given is the average of the individual spot perturbations determined from each orbit. Figure 4 therefore also shows the standard error in the mean for each Magsat spot estimate. Clearly, both the mean and standard error is then dependent on the number and orientation of tracks and the width of the longitude filter. $2.5^{\circ}$ was chosen here as a trade-off between the desire to maximize the number of Magsat spot values used in the calculation, i.e. the number of orbits through the ionospheric map, and the minimising of potential errors introduced by a widening sector of local time. There may be further work required here to determine if an optimum technique is determinable. However, the approach here at least allows some (initial) estimate of the possible spread in spot values.

Of the 19 observatories, Alert (ALE), Resolute (RES) and Thule (THL) are probably within the polar cap during active field conditions. Observatories at lower latitudes than about Eskdalemuir (ESK) or Meanook (MEA) are probably South of the location of any expanded auroral oval.

In the afternoon sector (i.e. Fig. 4) the Magsat model appears to reasonably characterize the observed perturbations within the polar cap and to the South of the auroral zone, in most components and at most observatories. Within the probable auroral oval itself (i.e. anywhere between DIK and ESK), there is a greater variability in both modelled and measured data, in terms of magnitudes and signs. This may reflect short-wavelength structures within the auroral oval as well as time-variations not captured by the averaging procedure implicit in the ionospheric maps. The tesseral scale and regularisation (see Appendix) may also be significant for short-wavelength features in the data.

It is noted that some observatory components may be 'anomalous', in particular $\delta B_{Y}$ at Irkutsk (IRK), which further complicates the comparison. There are clearly other factors that have not been accounted for here. We have subtracted an estimate of the crustal field component at the observatory, but not at the satellite (though the crustal field at the satellite should be a smaller proportion of the total field measured there). We have also not taken into account the contribution from field aligned currents and induced fields in the perturbations recorded at the observatory.

In the morning sector (Fig. 5) we note that the Magsat model does not provide spot values at a number of lower latitude observatories. This is a result of the longitude filter width ( \pm 2.5 degrees). In the polar cap, the model (BX has the 'wrong' sign, though with the correct magnitude. Otherwise the model seems to reflect the sign of the perturbation 

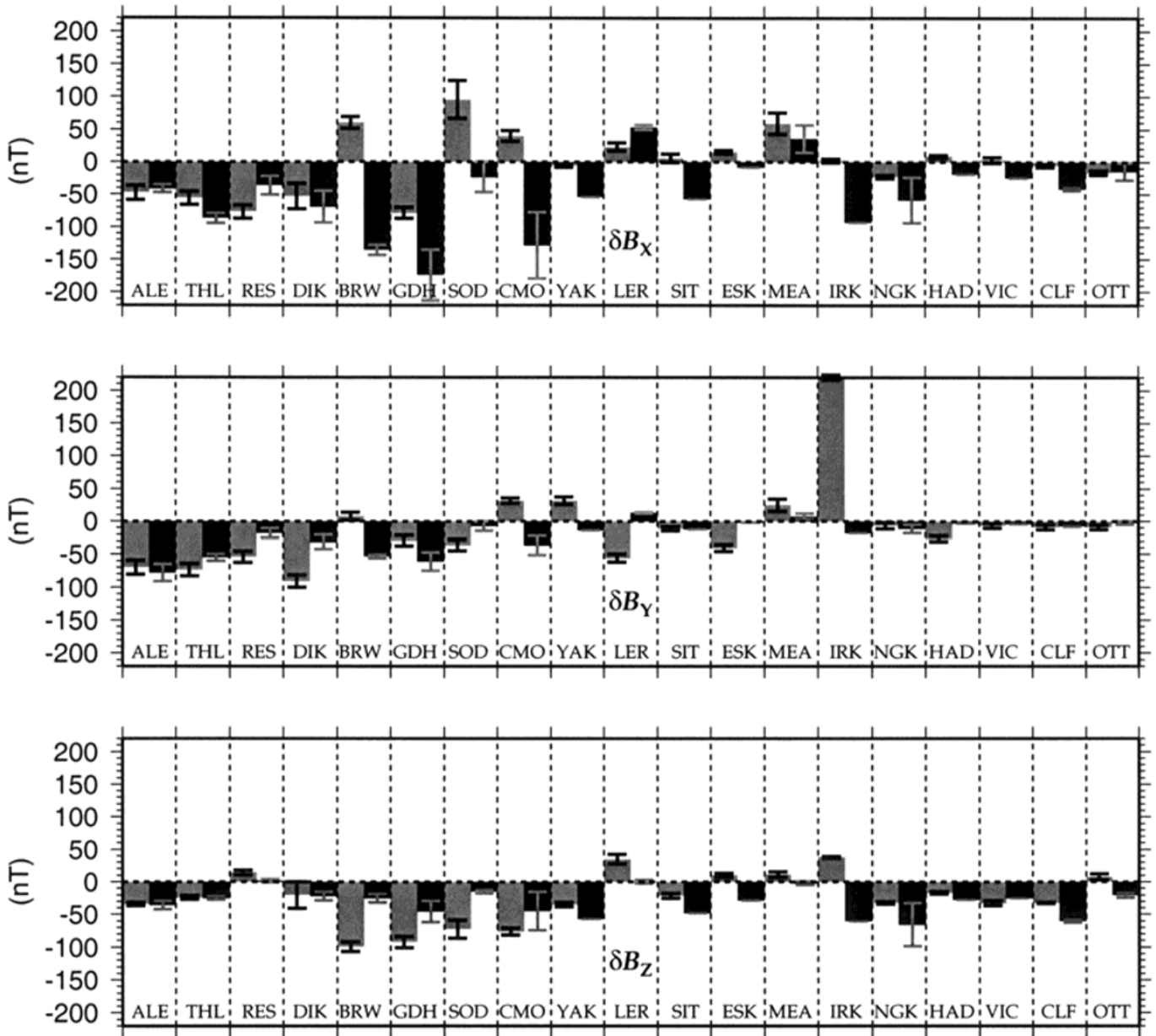

Fig. 4. Observatory hourly mean perturbations, of ionospheric origin, are shown in grey, with \pm 1 standard errors in the mean shown in black for clarity The equivalent Magsat ionospheric model spot values are shown in black, with \pm 1 standard errors in the mean shown in grey. The observatory data are averages of all hourly means during January 1980, when $K p \geq 4$, and at local times between 1200 and 2400 (i.e. local afternoon), with a main-field and crustal estimate subtracted. There were between 18 and 48 hourly means per observatory, depending on local conditions and between 1 and 11 Magsat spot values per observatory, depending on latitude (with more Magsat estimates for the higher latitude observatories). The observatories are ordered by geographic colatitude, left to right. Field perturbations are in geodetic North $(X)$, East $(Y)$ and vertically down $(Z)$ coordinates. IAGA observatory codes are shown.

reasonably well, at least in some components and at some observatories, though probably not as well as in the afternoon sector (i.e. Fig. 4).

Examinations of individual orbit data suggest that the tesseral currents physically closest to each observatory tend to dominate the perturbation calculated for that observatory. Thus there is the potential for a 'sensitivity' of the computed perturbation on the exact location and magnitude of nearby current elements, particularly when the current changes markedly between neighbouring tesserae, such as can be seen in the auroral zone of Fig. 2. This also suggests that the tesseral scale may be significant, and indeed one degree in latitude is over $100 \mathrm{~km}$ on the ground. If this is true then it may prove valuable to smooth the ionospheric maps, reducing the incidence of large steps in current between neighbouring elements. This needs to be examined further.

\section{Discussion and Conclusions}

The middle and high latitude field perturbation maps, i.e. Figs. 1 and 2 and their equivalents for the Southern hemi- sphere, show features which vary with external activity level and season and which are consistent with previous studies. The corresponding tessera-average current systems seem to provide a reasonable approximation to the exact horizontal ionospheric currents during the Magsat mission (as evidenced by Figs. 4 and 5). The results suggest that, at the lower activity levels that are of interest to main-field modelers, we may be able to use such ionospheric maps to provide a priori information on the average ionospheric contribution to measured magnetic data. However it has been emphasized a number of times that there may be more work required in order to determine the best use of these such maps.

The average currents are most accurate in quantifying the field perturbation parallel to the main-field at the satellite altitude, $\delta B_{\|}$. Thus these currents may provide a 'cleaned-up' field component for use in main-field modelling, i.e. by subtracting the model $\delta B_{\|}$from the measured data to leave an estimate of the core-generated component of $\delta B_{\|}$. Alternatively, the model $\delta B_{\|}$might be used as an initial constraint in iterative procedures that obtain a combined main-field and ionospheric model from satellite measurements. Either way, 

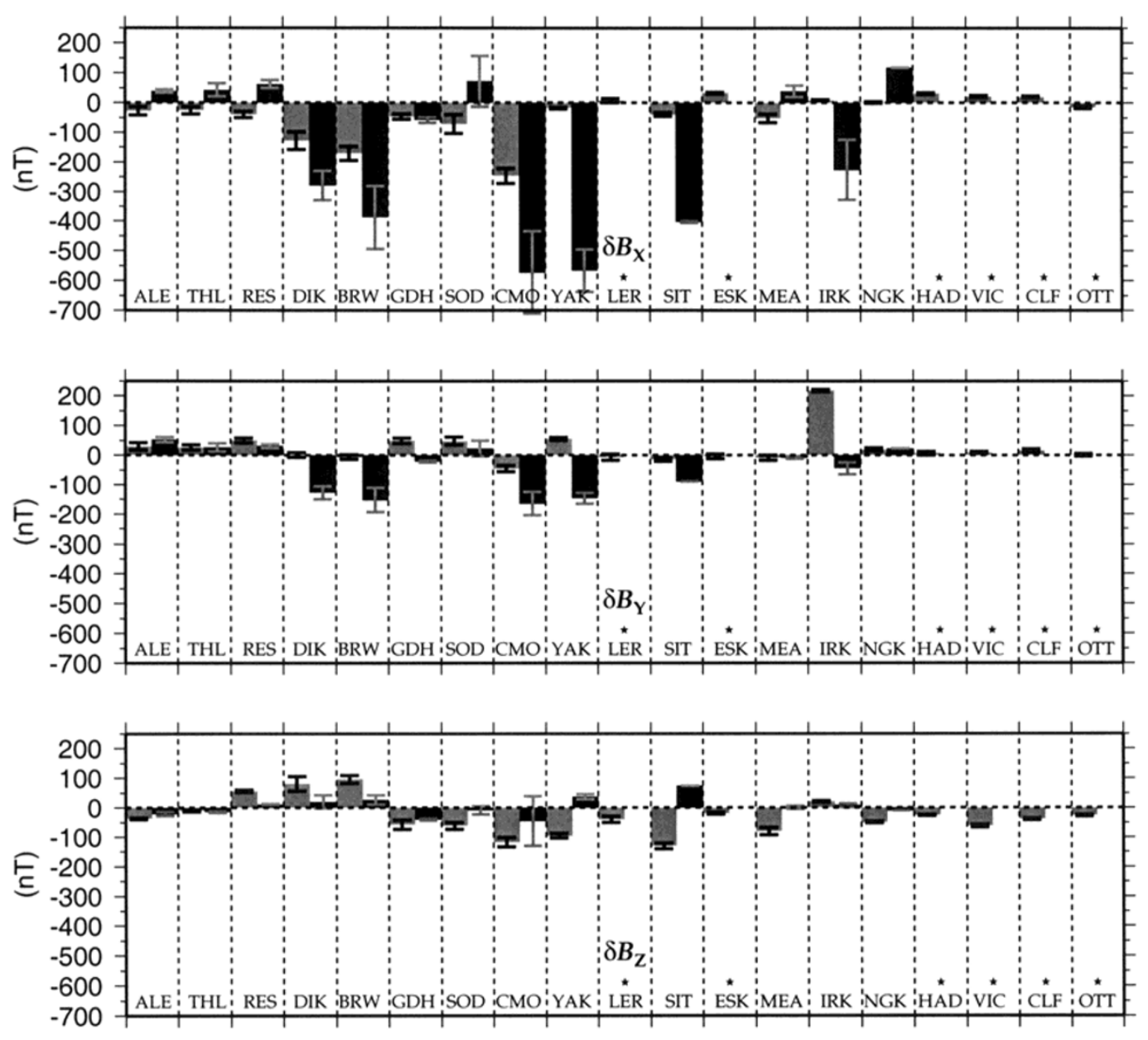

Fig. 5. As Fig. 4, but for local times between 0000 and 1200 (i.e. local morning). Asterisks denote observatories for which no model values are available (i.e. where there were no Magsat orbits within \pm 2.5 degrees in longitude, at the latitude of the observatory).

it should be noted, we need an existing, if only approximate, main-field model to estimate the local field inclination (as discussed in the Appendix).

Improving the accuracy of the computed perturbation in the plane for which the field line is a normal (e.g. $\delta B_{\perp}$ ) and, more generally, the magnetic field of field-aligned currents, will require a different approach. The toroidal-poloidal decomposition method described by Backus (1986) seems appropriate here.

The 'ground-truth' aspect of the study shows that these ionospheric maps broadly reproduce the observed amplitudes and display some consistency in the signs of the observatory perturbations, at least outwith the auroral oval. This suggests that they are physically sensible and that the ionospheric current contribution to the fields measured at the satellite has been fairly well characterised. We have suggested that within the auroral zone the tessera dimension becomes significant. Otherwise one suspects that the rapid time variations in fields and currents, particularly during active periods, have not been adequately captured by the technique.

Although there are differences in satellite altitude and solar cycle phase between Magsat and Ørsted, Ørsted data should provide an opportunity to check the consistency, mission to mission, of the average maps derived here. Indeed we regard the work described here as an initial step towards exploiting the Ørsted data set. Some attempt has been made to use the 1991-1993 Polar Orbiting Geomagnetic Survey (POGS) satellite mission data as an intermediate stage. However we believe that the lack of truly oriented vector data and the calibration drifts in this data set make our equivalent POGS results inconclusive. This may be investigated further in the future to provide a 'bridge' in time between Magsat and Ørsted.

Ørsted, if successful, should also provide the opportunity to extend the maps to all MLT and seasons. Such maps can then be used, as described above, in the production of mainfield models. These, in turn, can be compared with models produced in more 'traditional' ways; for example those procedures which neglect available high-latitude satellite vector measurements.

The expected density of Ørsted data may also allow an easier comparison with other approaches to quantifying the ionospheric field, on both a 'snapshot' basis as well as the longer-time, statistical average, basis considered here.

Acknowledgments. The work presented here developed from early studies undertaken along with David Barraclough, Toby Clark and Susan Macmillan of the British Geological Survey. I would therefore like to acknowledge their comments and practical help. I 
would also like to thank Therese Moretto for her comments, which have helped to improve the clarity of the paper. This paper is published with the permission of the Director, British Geological Survey (NERC). This work was supported by a UK 'Realising Our Potential Award', ROPA grant number GR3/R9537.

\section{Appendix}

Olsen's method (Olsen, 1996) involves the subtraction of a main-field estimate $\mathbf{B}_{0}$ (here the GSFC (12/83) model of Langel and Estes, 1985b) and a large-scale magnetospheric field $\mathbf{B}_{\text {mag }}$ (Langel and Estes, 1985a) from the measured satellite field, $\mathbf{B}_{\mathrm{obs}}$, at each measurement point along an orbit. The residual magnetic vector is then $\delta B=\left(\delta B_{R}, \delta B_{H}, \delta B_{D}\right)$, in terms of field residuals in the radial direction, in the horizontal direction in the meridional plane of the main field and in the direction perpendicular to the meridional plane.

These residual fields can be decomposed into $\delta B_{\|}$and $\delta B_{\perp}$, parallel and perpendicular to the field in the meridional plane of the main field, using $\delta B_{R}, \delta B_{H}$ and the calculated main field inclination at the satellite observation point. Olsen's method then assumes that $\delta B_{R}$ and $\delta B_{H}$ are generated by a set of infinite line currents perpendicular to the orbital plane, i.e. approximately East-West for close to polar-orbiting satellites, such as Magsat. These line currents are located at a common radial distance from the Earth's centre and at regular intervals along a track beneath the satellite orbit, in the ionosphere. It is also assumed that this track is in the meridional plane of the main field.

The individual current strengths are determined from the well-known expression for the field of a line current, using the geometrical relationship between the position of each line current element and each satellite measurement along the orbit, as illustrated in Olsen (1996, figure 1).

The result is a matrix expression for each orbit, $\mathbf{y}=$ $\mathbf{G} \cdot \mathbf{x}$, which relates the measurement data vector $\mathbf{y}=\left(\delta B_{\|, 1}\right.$, $\left.\delta B_{\|, 2}, \ldots, \delta B_{\|, N}\right)$ at $N$ points along the orbit and the current vector $\mathbf{x}=\left(J_{1}, J_{2}, \ldots, J_{M}\right)$ of $M$ line currents for that orbit. The elements of the matrix $\mathbf{G}$ are determined from a regularised least squares solution (ridge-regression) given by Marquardt (1970). Note that $\delta B_{\|}$is preferred in the solution as this component has, to a first approximation, no contribution from neighbouring field-aligned currents.

We define the common current radius as $6371+115 \mathrm{~km}$, with a separation between line currents, i.e. along the "current orbit', of about $113 \mathrm{~km}$ (corresponding to an angle subtended at the centre of the Earth of $1^{\circ}$ ).

The method allows for the solution for the current vectors $\mathbf{x}=\left(J_{1}, J_{2}, \ldots, J_{M}\right)$ for each orbit in the satellite data set (November 1979 to May 1980). To allow for some smoothing, the Magsat data set has been reduced to a sequence of 5 -second samples by along-track averaging of the original 0.5 second data. Discontinuities which are known to exist in the measured data (caused by Magsat attitude changes, see Langel and Hinze, 1998) have not been treated in this analysis. However the time-averaging process should go some way to reducing the significance of this error.
Average ionospheric current maps can then be calculated according to various filterings of the Magsat data, for example, by season, magnetic local time, $K p$ and $D s t$ indices. These filters are typical of the treatment of data in main-field modelling. More selective filtering, e.g. by IMF $B_{y}$ and $B_{z}$, is found to markedly reduce the sample sizes and is therefore not considered here, but may be of greater use with a larger data set, such as may be provided by Ørsted. Line currents are calculated for all Magsat orbit segments with geocentric colatitudes between $0^{\circ}$ and $50^{\circ}$ (i.e. North) and between $130^{\circ}$ and $180^{\circ}$ (South).

To construct each map, the North and South hemispheres are each divided into 20,626 equal-area tesserae, with the area of each tessera equal to that of a $1^{\circ} \times 1^{\circ}$ dipole-latitude/MLT sector at the equator. The average ionospheric currents are then derived by binning the exact, i.e. model solution, currents for each of the filtered orbits according to tesseral number, that is, by dipole-latitude/MLT. Tessera-average field perturbations are also calculated by the same binning procedure.

\section{References}

Backus, G., Poloidal and toroidal fields in geomagnetic field modelling, Rev. Geophys., 24, 75-109, 1986.

Kamide, Y. and S. Matsushita, Simulation studies of ionospheric electric fields and currents in relation to field-aligned currents: 1 . quiet periods J. Geophys. Res., 84, 4083-4098, 1979a.

Kamide, Y. and S. Matsushita, Simulation studies of ionospheric electric fields and currents in relation to field-aligned currents: 2 . substorms, $J$. Geophys. Res., 84, 4099-4115, 1979b.

Kamide, Y., A. D. Richmond, and S. Matsushita, Estimation of ionospheric electric fields, ionospheric currents, and field-aligned currents from ground magnetic records, J. Geophys. Res., 86, 801-813, 1981.

Kisabeth, J. L. and G. Rostoker, Modelling of three-dimensional current systems associated with magnetospheric substorms, Geophys. J. R. Astr Soc., 49, 655-683, 1977.

Langel, R. A., The main field, in Geomagnetism, Vol. 1 Ch. 4, edited by J. A. Jacobs, pp. 249-512, Academic Press, London, 1987.

Langel, R. A. and R. H. Estes, Large-scale, near-earth magnetic fields from external sources and the corresponding induced internal field, J. Geophys. Res., 90, 2487-2494, 1985a.

Langel, R. A. and R. H. Estes, The near-earth magnetic field at 1980 determined from MAGSAT data, J. Geophys. Res., 90, 2495-2510, 1985b.

Langel, R. A. and W. J. Hinze, The magnetic field of the earth's lithosphere, Cambridge University Press, 1998.

Marquardt, D. W., Generalized inverses, ridge regression, biased linear estimation and non-linear estimation, Technometrics, 12, 591-612, 1970.

Olsen, N., A new tool for determining ionospheric currents from magnetic satellite data, Geophys. Res. Lett., 23, 3635-3638, 1996.

Quinn, J. M., R. J. Coleman, S. Macmillan, and D. R. Barraclough, The 1995 revision of the joint US/UK geomagnetic field models. II: Main Field, J. Geomag. Geoelectr., 49, 245-261, 1997.

Rich, F. J. and Y. Kamide, Convection electric fields and ionospheric currents derived from model field-aligned currents at high latitudes, J. Geophys. Res., 88, 271-281, 1983.

Thomson, A. W. P., S. Macmillan, and D. R. Barraclough, Geomagnetic main-field modelling with POGS satellite data, J. Geomag. Geoelectr., 49, 417-440, 1997.

Walker, J. K., V. Y. Semenov, and T. L. Hansen, Synoptic models of high latitude magnetic activity and equivalent ionospheric and induced currents, J. Atmos. Solar-Terr. Phys., 59, 1435-1452, 1997.

A. W. P. Thomson (e-mail: a.thomson@bgs.ac.uk) 